\title{
Magnetic-field dependence of the oxygen isotope effect on the magnetic penetration depth of hole-doped cuprate superconductors
}

\author{
R. Khasanov, ${ }^{1, *}$ A. Shengelaya, ${ }^{1,2}$ D. Di Castro, ${ }^{1,3}$ D. G. Eshchenko, ${ }^{1,4}$ I. M. Savić, ${ }^{5}$ K. Conder, ${ }^{6}$ E. Pomjakushina, ${ }^{6,7}$ \\ J. Karpinski, ${ }^{8}$ S. Kazakov, ${ }^{8}$ and H. Keller ${ }^{1}$ \\ ${ }^{1}$ Physik-Institut der Universität Zürich, Winterthurerstrasse 190, CH-8057 Zürich, Switzerland \\ ${ }^{2}$ Physics Institute of Tbilisi State University, Chavchavadze 3, GE-0128 Tbilisi, Georgia \\ ${ }^{3}$ CNR-INFM-Coherentia and Dipartimento di Fisica, Universita di Roma La Sapienza, Piazzale Aldo Moro 2, I-00185 Roma, Italy \\ ${ }^{4}$ Laboratory for Muon Spin Spectroscopy, Paul Scherrer Institut, CH-5232 Villigen PSI, Switzerland \\ ${ }^{5}$ Faculty of Physics, University of Belgrade, 11001 Belgrade, Serbia \\ ${ }^{6}$ Laboratory for Developments and Methods, Paul Scherrer Institut, CH-5232 Villigen PSI, Switzerland \\ ${ }^{7}$ Laboratory for Neutron Scattering, ETH Zürich and Paul Scherrer Institut, CH-5232 Villigen PSI, Switzerland \\ ${ }^{8}$ Solid State Physics Laboratory, ETH 8093 Zürich, Switzerland \\ (Received 18 October 2006; revised manuscript received 14 December 2006; published 21 February 2007)
}

\begin{abstract}
The magnetic-field dependence of the oxygen isotope $\left({ }^{16} \mathrm{O} /{ }^{18} \mathrm{O}\right)$ effect $(\mathrm{OIE})$ on the in-plane magnetic-field penetration depth $\lambda_{a b}$ was studied in the hole-doped high-temperature cuprate superconductors $\mathrm{YBa}_{2} \mathrm{Cu}_{4} \mathrm{O}_{8}$, $\mathrm{Y}_{0.8} \mathrm{Pr}_{0.2} \mathrm{Ba}_{2} \mathrm{Cu}_{3} \mathrm{O}_{7-\delta}$, and $\mathrm{Y}_{0.7} \mathrm{Pr}_{0.3} \mathrm{Ba}_{2} \mathrm{Cu}_{3} \mathrm{O}_{7-\delta}$. It was found that the OIE on $\lambda_{a b}$ decreases by more than a factor of 2 with increasing magnetic field from $\mu_{0} H=0.2 \mathrm{~T}$ to $\mu_{0} H=0.6 \mathrm{~T}$. This effect can be explained by the isotope dependence of the in-plane charge carrier mass $m_{a b}^{*}$.
\end{abstract}

DOI: 10.1103/PhysRevB.75.060505

PACS number(s): 74.25.Ha, 74.72.Bk, 76.75.+i

The presence of nodes in the superconducting gap is probably one of the most significant features of high-temperature cuprate superconductors (HTS's) which has attracted considerable theoretical and experimental attention in recent years (see, e.g., Refs. 1 and 2). The fourfold-symmetric nature of the $d$-wave order parameter, together with the presence of gap nodes on the Fermi surface, opens possibilities for novel effects to be observable in HTS's. One of the most remarkable effects is the magnetic-field dependence of the in-plane magnetic penetration depth $\lambda_{a b}$ observed in various HTS's in the mixed state (see, e.g., Ref. 3 and references therein). It was shown that the field-dependent correction to $\lambda_{a b}$ arises from the nonlocal and nonlinear response of a superconductor to an applied magnetic field. ${ }^{4,5}$ In contrast to conventional extreme type-II BCS superconductors, both nonlinear and nonlocal corrections to $\lambda_{a b}$ were found to be very strong in HTS's. ${ }^{3-5}$

The nonlinear correction to $\lambda_{a b}$ arises from magneticfield-induced quasiparticle excitation in the gap nodes. ${ }^{6}$ The excitation energy $\varepsilon$ is associated with the pair momentum $2 \mathbf{q}=m^{*} \mathbf{v}_{s}\left(\mathbf{v}_{s}\right.$ is the local superfluid velocity, and $m^{*}$ is the charge carrier mass) and the Fermi velocity $\mathbf{v}_{F}$ (Ref. 7):

$$
\varepsilon=\mathbf{v}_{F} \cdot \mathbf{q} \text {. }
$$

The density of the delocalized states was found to increase proportionally to $\sqrt{H}$. ${ }^{6}$ The nonlocal correction to $\lambda$ appears from the response of electrons with momenta on the Fermi surface close to the gap nodes. This is because the coherence length $\xi$, being inversely proportional to the gap, becomes very large close to the nodes and formally diverges at the nodal points. Thus, there exist areas on the Fermi surface where $\lambda / \xi \lesssim 1$ and the response of a superconductor to an applied magnetic field becomes nonlocal. ${ }^{5}$

A simple analysis reveals that both the nonlinear and nonlocal corrections depend on the supercarrier mass $m^{*}$. Indeed, the nonlinear correction to $\lambda_{a b}$ depends on the excitation en$\operatorname{ergy} \varepsilon$ [see Eq. (1)] and, therefore, will increase with increasing $\varepsilon$. Bearing in mind that $\left|\mathbf{v}_{F}\right| \propto 1 / m^{*}$ and $\left|\mathbf{v}_{s}\right| \propto 1 / m^{*},{ }^{6}$ the excitation energy is proportional to $1 / \mathrm{m}^{*}$. Thus the nonlinear correction to $\lambda_{a b}$ decreases with increasing supercarrier mass $m^{*}$. On the other hand, the nonlocal correction depends on the Fermi surface area where, due to the growing of $\xi$ close to the nodes, $\lambda / \xi \lesssim 1 .^{5}$ Therefore, the nonlocal correction will decrease with increasing Ginzburg-Landau parameter $\kappa=\lambda_{a b} / \xi_{0}$. Taking into account that within the simple London model $\lambda^{-2} \propto 1 / m^{*}$ and $\xi_{0} \propto\left|\mathbf{v}_{F}\right| / \Delta_{0}$, one can easily get $\kappa \propto\left(m^{*}\right)^{3 / 2}\left(\xi_{0}\right.$ and $\Delta_{0}$ are the mean values of the coherence length and the superconducting gap, respectively). This implies that the nonlocal correction to $\lambda_{a b}$, in full analogy with

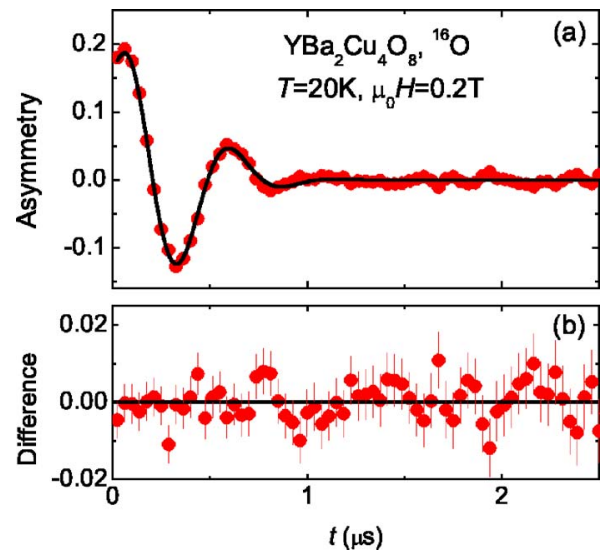

FIG. 1. (Color online) (a) TF- $\mu$ SR spectra in ${ }^{16} \mathrm{O}$-substituted $\mathrm{YBa}_{2} \mathrm{Cu}_{4} \mathrm{O}_{8}$ sample in a magnetic field of $0.2 \mathrm{~T}$ at temperature $T$ $=20 \mathrm{~K}$. The solid line represents fit with the Gaussian relaxation function $R(t)=\exp \left[-\sigma^{2} t^{2} / 2\right]$. For visualization purposes the apparent precession frequencies are modified from the actual precession frequencies by the use of a rotating reference frame. (b) Difference between the experimental data and the fit. 

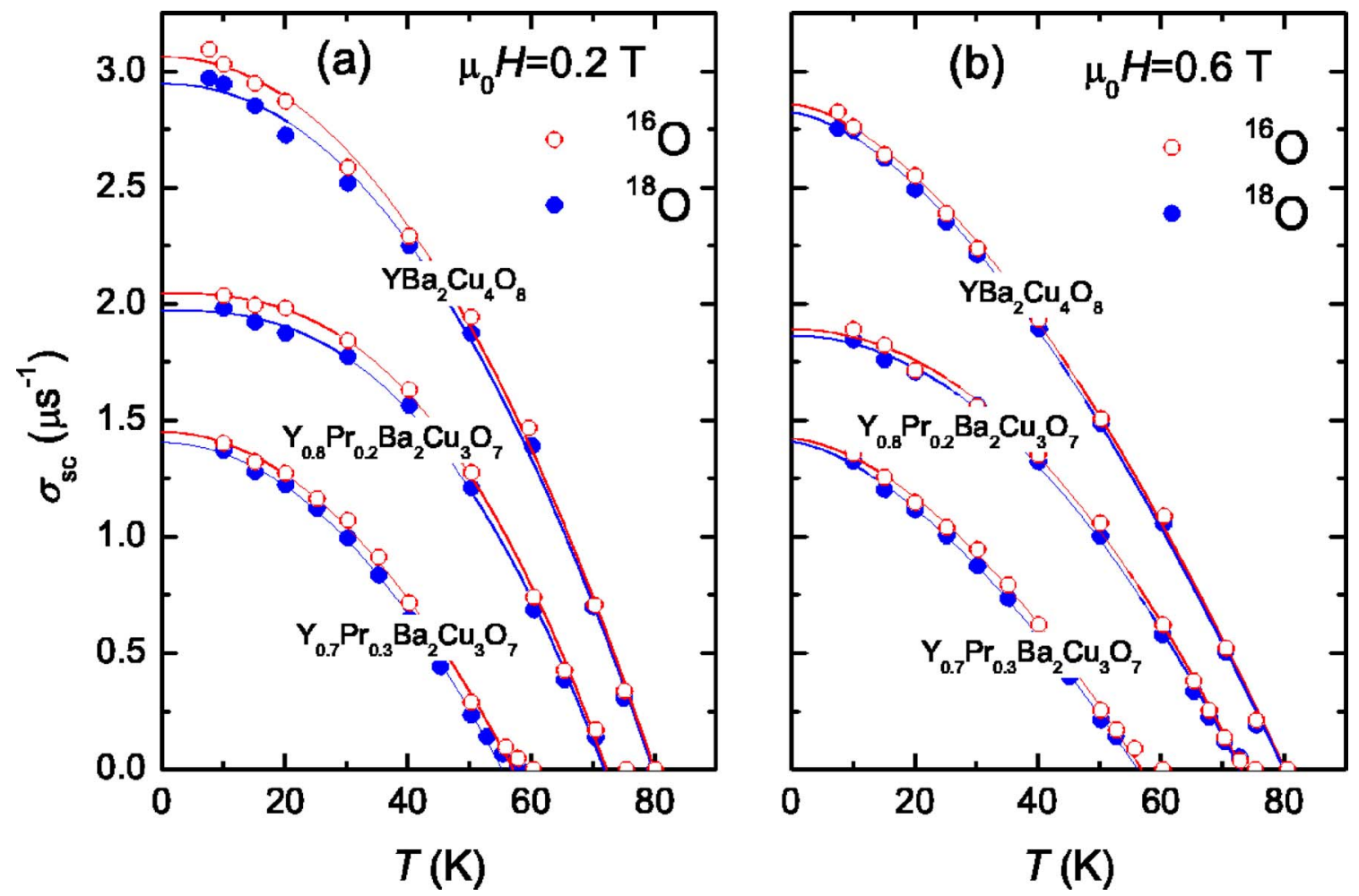

FIG. 2. (Color online) Temperature dependences of the superconducting part of the $\mu$ SR depolarization rate $\sigma_{s c}$ of ${ }^{16} \mathrm{O}-(\bigcirc)$ and ${ }^{18} \mathrm{O}-(\bullet)$ substituted $\mathrm{YBa}_{2} \mathrm{Cu}_{4} \mathrm{O}_{8}, \mathrm{Y}_{0.8} \mathrm{Pr}_{0.2} \mathrm{Ba}_{2} \mathrm{Cu}_{3} \mathrm{O}_{7-\delta}$, and $\mathrm{Y}_{0.7} \mathrm{Pr}_{0.3} \mathrm{Ba}_{2} \mathrm{Cu}_{3} \mathrm{O}_{7-\delta}$. (a) $\mu_{0} H=0.2 \mathrm{~T}$. (b) $\mu_{0} H=0.6 \mathrm{~T}$. The errors are smaller than the size of the data points. The solid lines correspond to fits to the power law $\sigma_{s c}(T) / \sigma_{s c}(0)=1-\left(T / T_{c}\right)^{n}$ with parameters listed in Table I. $\sigma_{s c}$ vs $T$ curves at $\mu_{0} H=0.2 \mathrm{~T}$ for ${ }^{16} \mathrm{O} /{ }^{18} \mathrm{O}$-substituted $\mathrm{YBa}_{2} \mathrm{Cu}_{4} \mathrm{O}_{8}$ and $\mathrm{Y}_{0.8} \mathrm{Pr}_{0.2} \mathrm{Ba}_{2} \mathrm{Cu}_{3} \mathrm{O}_{7-\delta}$ samples are reproduced from $\mathrm{Ref}$. 13 .

the nonlinear one, decreases with increasing $m^{*}$. As a consequence, the magnetic-field dependence of $\lambda_{a b}$ is expected to be stronger for a superconductor with a smaller $m^{*}$.

A study of the field dependence of the isotope effect on $\lambda_{a b}$ can serve as a direct test of this prediction. Indeed, several oxygen isotope $\left({ }^{16} \mathrm{O} /{ }^{18} \mathrm{O}\right)$ effect (OIE) studies of the in-plane penetration depth $\lambda_{a b}$ in various cuprate families $\left[\mathrm{Y}_{1-x} \mathrm{Pr}_{x} \mathrm{Ba}_{2} \mathrm{Cu}_{3} \mathrm{O}_{7-\delta}\right.$ (Refs. 8-13), $\mathrm{YBa}_{2} \mathrm{Cu}_{4} \mathrm{O}_{8}$ (Ref. 13), $\mathrm{La}_{2-x} \mathrm{Sr}_{x} \mathrm{CuO}_{4}$ (Refs. 12-16), and $\mathrm{Bi}_{1.6} \mathrm{~Pb}_{0.4} \mathrm{Sr}_{2} \mathrm{Ca}_{2} \mathrm{Cu}_{3} \mathrm{O}_{10+\delta}$ (Ref. 17)] showed a pronounced oxygen-mass dependence of the in-plane supercarrier mass with ${ }^{18} m_{a b}^{*}>{ }^{16} m_{a b}^{*}$. This implies that ${ }^{16} \lambda_{a b}$ should increase stronger with magnetic field than ${ }^{18} \lambda_{a b}$. Bearing in mind that ${ }^{18} \lambda_{a b}$ is always larger than ${ }^{16} \lambda_{a b}$ (Refs. 8-18) the OIE on $\lambda_{a b}\left[\Delta \lambda_{a b} / \lambda_{a b}=\left({ }^{18} \lambda_{a b}\right.\right.$ $\left.\left.-{ }^{16} \lambda_{a b}\right) /{ }^{16} \lambda_{a b}\right]$ should decrease with increasing magnetic field (hereafter the indices 16 and 18 denote ${ }^{16} \mathrm{O}$ and ${ }^{18} \mathrm{O}$ substituted samples, respectively).

Here, we report a study of the magnetic-field dependence of the OIE on $\lambda_{a b}$ in $\mathrm{YBa}_{2} \mathrm{Cu}_{4} \mathrm{O}_{8}$ and $\mathrm{Y}_{1-x} \mathrm{Pr}_{x} \mathrm{Ba}_{2} \mathrm{Cu}_{3} \mathrm{O}_{7-\delta}$ $(x=0.2,0.3)$ by means of the transverse-field muon-spin rotation (TF- $\mu \mathrm{SR}$ ) technique. The isotope shift of $\lambda_{a b}$ at $T=0$ was found to decrease from $\Delta \lambda_{a b} / \lambda_{a b}=2.2(6) \%, 2.4(4) \%$, and $2.1(6) \%$ at $\mu_{0} H=0.2 \mathrm{~T}$ to $0.9(6) \%, \quad 1.0(4) \%$, and $0.9(6) \%$ at $\mu_{0} H=0.6 \mathrm{~T}$ for $\mathrm{YBa}_{2} \mathrm{Cu}_{4} \mathrm{O}_{8}$, $\mathrm{Y}_{0.8} \mathrm{Pr}_{0.2} \mathrm{Ba}_{2} \mathrm{Cu}_{3} \mathrm{O}_{7-\delta}$, and $\mathrm{Y}_{0.7} \mathrm{Pr}_{0.3} \mathrm{Ba}_{2} \mathrm{Cu}_{3} \mathrm{O}_{7-\delta}$, respectively. Moreover, measurements of the magnetic-field dependence of $\lambda_{a b}$ at $T=20 \mathrm{~K}$ for $\mathrm{YBa}_{2} \mathrm{Cu}_{4} \mathrm{O}_{8}$ reveal that for $\mu_{0} H$ $\gtrsim 0.2 \mathrm{~T}$, the penetration depth $\lambda_{a b}$ increases more strongly for the ${ }^{16} \mathrm{O}$-substituted sample than for the ${ }^{18} \mathrm{O}$-substituted one, consistent with the finding ${ }^{18} m_{a b}^{*}>{ }^{16} m_{a b}^{*}$.

Details on the sample preparation for $\mathrm{YBa}_{2} \mathrm{Cu}_{4} \mathrm{O}_{8}$ and $\mathrm{Y}_{1-x} \mathrm{Pr}_{x} \mathrm{Ba}_{2} \mathrm{Cu}_{3} \mathrm{O}_{7-\delta}(x=0.2,0.3)$ can be found elsewhere. ${ }^{19,20}$ Oxygen isotope exchange was performed during heating the samples in ${ }^{18} \mathrm{O}_{2}$ gas. In order to ensure that the ${ }^{16} \mathrm{O}$ - and ${ }^{18} \mathrm{O}$-substituted samples are subject of the same thermal history, the annealing of the two samples was performed simultaneously in ${ }^{16} \mathrm{O}_{2}$ and ${ }^{18} \mathrm{O}_{2}$ (95\%-enriched) gas, respectively. ${ }^{20}$ The ${ }^{18} \mathrm{O}$ content in all the samples, as determined from a change of the sample weight after the isotope exchange, was found to be $82(2) \%$.

The TF- $\mu \mathrm{SR}$ experiments were performed at the Paul Scherrer Institute (PSI), Switzerland, using the $\pi \mathrm{M} 3 \mu \mathrm{SR}$ facility. In a powder sample the magnetic penetration depth $\lambda$ can be extracted from the muon-spin depolarization rate $\sigma(T) \propto 1 / \lambda^{2}(T)$, which probes the second moment $\left\langle\Delta B^{2}\right\rangle$ of the probability distribution of the local magnetic-field function $p(B)$ in the mixed state. ${ }^{21}$ For highly anisotropic layered superconductors (like cuprate superconductors) $\lambda$ is mainly determined by the in-plane penetration depth $\lambda_{a b}$ (Ref. 21): $\sigma(T) \propto 1 / \lambda_{a b}^{2}(T)$. The depolarization rate $\sigma$ was extracted from the $\mu$ SR time spectra using a Gaussian relaxation function $R(t)=\exp \left[-\sigma^{2} t^{2} / 2\right]$ (see Fig. 1). The superconducting contribution $\sigma_{s c}$ was then obtained by subtracting the dipolar contribution $\sigma_{n m}$ measured above $T_{c}$ as $\sigma_{s c}^{2}=\sigma^{2}-\sigma_{n m}^{2}$.

Figure 2 shows the temperature dependences of $\sigma_{s c}$ for the samples studied in this work, measured after field-cooling the samples from far above $T_{c}$ in $\mu_{0} H=0.2 \mathrm{~T}$ (a) and $0.6 \mathrm{~T}$ (b). In both fields $\sigma_{s c}$ for the ${ }^{18} \mathrm{O}$-substituted samples is sys- 
TABLE I. Summary of the OIE results for $\mathrm{YBa}_{2} \mathrm{Cu}_{4} \mathrm{O}_{8}, \mathrm{Y}_{0.8} \mathrm{Pr}_{0.2} \mathrm{Ba}_{2} \mathrm{Cu}_{3} \mathrm{O}_{7-\delta}$, and $\mathrm{Y}_{0.7} \mathrm{Pr}_{0.3} \mathrm{Ba}_{2} \mathrm{Cu}_{3} \mathrm{O}_{7-\delta}$ extracted from the experimental data (see text for an explanation). The values of $\Delta \lambda_{a b}(0, H) / \lambda_{a b}(0, H)$ were corrected for incomplete ${ }^{18} \mathrm{O}$ exchange of $82 \%$.

$$
\mu_{0} H=0.2 \mathrm{~T}
$$

$\mu_{0} H=0.6 \mathrm{~T}$

\begin{tabular}{|c|c|c|c|c|c|c|c|c|c|c|}
\hline Sample & $\begin{array}{l}{ }^{16} T_{c} \\
{[\mathrm{~K}]}\end{array}$ & $\begin{array}{c}{ }^{16} \sigma_{s c}(0) \\
{\left[\mu \mathrm{s}^{-1}\right]}\end{array}$ & $\begin{array}{l}{ }^{18} T_{c} \\
{[\mathrm{~K}]}\end{array}$ & $\begin{array}{c}{ }^{18} \sigma_{s c}(0) \\
{\left[\mu \mathrm{s}^{-1}\right]}\end{array}$ & $\frac{\Delta \lambda_{a b}(0)}{\lambda_{a b}(0)}$ & $\begin{array}{l}{ }^{16} T_{c} \\
{[\mathrm{~K}]}\end{array}$ & $\begin{array}{c}{ }^{16} \sigma_{s c}(0) \\
{\left[\mu \mathrm{s}^{-1}\right]}\end{array}$ & $\begin{array}{l}{ }^{18} T_{c} \\
{[\mathrm{~K}]}\end{array}$ & $\begin{array}{c}{ }^{18} \sigma_{s c}(0) \\
{\left[\mu \mathrm{s}^{-1}\right]}\end{array}$ & $\frac{\Delta \lambda_{a b}(0)}{\lambda_{a b}(0)}$ \\
\hline $\mathrm{YBa}_{2} \mathrm{Cu}_{4} \mathrm{O}_{8}$ & $80.03(45)$ & $3.06(2)$ & $79.77(45)$ & $2.95(2)$ & $2.2(6)$ & $80.17(37)$ & $2.86(2)$ & 79.99(37) & $2.82(2)$ & $0.9(6)$ \\
\hline $\mathrm{Y}_{0.8} \mathrm{Pr}_{0.2} \mathrm{Ba}_{2} \mathrm{Cu}_{3} \mathrm{O}_{7-\delta}$ & $72.22(38)$ & $2.05(1)$ & $71.82(44)$ & $1.97(1)$ & $2.4(4)$ & $73.19(35)$ & $1.89(1)$ & $72.84(34)$ & $1.86(1)$ & $1.0(4)$ \\
\hline $\mathrm{Y}_{0.7} \mathrm{Pr}_{0.3} \mathrm{Ba}_{2} \mathrm{Cu}_{3} \mathrm{O}_{7-\delta}$ & $57.05(57)$ & $1.45(1)$ & $55.23(34)$ & $1.40(1)$ & $2.1(6)$ & $56.88(39)$ & $1.42(1)$ & $56.21(31)$ & $1.40(1)$ & $0.9(6)$ \\
\hline
\end{tabular}

tematically lower than those for the ${ }^{16} \mathrm{O}$-substituted ones. The data in Fig. 2 were fitted with the power law $\sigma_{s c}(T) / \sigma_{s c}(0)=1-\left(T / T_{c}\right)^{n}\left(\right.$ Ref. 21) with $\sigma_{s c}(0), n$, and $T_{c}$ as free parameters. The values of $T_{c}$ and $\sigma_{s c}(0)$, obtained from the fits, are listed in Table I. From $\sigma_{s c}(0)$ the relative isotope shift of the in-plane penetration depth $\Delta \lambda_{a b}(0) / \lambda_{a b}(0)=$ $-0.5\left[{ }^{18} \sigma_{s c}(0)-{ }^{16} \sigma_{s c}(0)\right] /{ }^{16} \sigma_{s c}(0)$ was determined (see Table I). The values of $\Delta \lambda_{a b}(0) / \lambda_{a b}(0)$ were corrected for the incomplete ${ }^{18} \mathrm{O}$ content $(82 \%)$ of the ${ }^{18} \mathrm{O}$-substituted samples.

Two important points should be considered: (i) For all the samples (except ${ }^{18} \mathrm{O}$-substituted $\mathrm{Y}_{0.7} \mathrm{Pr}_{0.3} \mathrm{Ba}_{2} \mathrm{Cu}_{2} \mathrm{O}_{7-\delta}$ ) $\sigma_{s c}(0)$ decreases with increasing magnetic field. The changes of $\sigma_{s c}(0)$ for both oxygen isotopes were found to be $\left[\sigma_{s c}(0.6 \mathrm{~T})-\sigma_{s c}(0.2 \mathrm{~T})\right] / \sigma_{s c}(0.2 \mathrm{~T}) \simeq-5 \%, \simeq-6 \%$, and $\simeq-1 \%$ for $\quad \mathrm{YBa}_{2} \mathrm{Cu}_{4} \mathrm{O}_{8}, \quad \mathrm{Y}_{0.8} \mathrm{Pr}_{0.2} \mathrm{Ba}_{2} \mathrm{Cu}_{3} \mathrm{O}_{7-\delta}, \quad$ and $\mathrm{Y}_{0.7} \mathrm{Pr}_{0.3} \mathrm{Ba}_{2} \mathrm{Cu}_{3} \mathrm{O}_{7-\delta}$, respectively. Note that the values for $\mathrm{YBa}_{2} \mathrm{Cu}_{4} \mathrm{O}_{8}$ and $\mathrm{Y}_{0.8} \mathrm{Pr}_{0.2} \mathrm{Ba}_{2} \mathrm{Cu}_{3} \mathrm{O}_{7-\delta}$ are in fair agreement with those reported in the literature for optimally doped $\mathrm{YBa}_{2} \mathrm{Cu}_{3} \mathrm{O}_{7-\delta}$ and $\mathrm{La}_{1.85} \mathrm{Sr}_{0.15} \mathrm{CuO}_{4} \cdot{ }^{3}$ The value of $-1 \%$ obtained for $\mathrm{Y}_{0.7} \mathrm{Pr}_{0.3} \mathrm{Ba}_{2} \mathrm{Cu}_{3} \mathrm{O}_{7-\delta}$ is somehow small. The reason for that is probably the enhancement of the magnetic contribution to the muon relaxation rate $\sigma$ at higher fields, observed in similar samples below $\sim 10 \mathrm{~K} .{ }^{9}$ (ii) In both fields ${ }^{16} \sigma_{s c}(0)>{ }^{18} \sigma_{s c}(0)\left[{ }^{16} \lambda_{a b}(0)<{ }^{18} \lambda_{a b}(0)\right]$. Bearing in mind that the density of the normal-state carriers for ${ }^{16} \mathrm{O}$ and ${ }^{18} \mathrm{O}$ samples studied in the present work were found to be the same, ${ }^{9,13}$ and assuming that at zero temperature and zero magnetic field all the carriers condense into Cooper pairs (in analogy with what was observed in Ref. 22), the in-plane charge carriers are expected to be heavier in the ${ }^{18} \mathrm{O}$-substituted samples than in the ${ }^{16} \mathrm{O}$-substituted ones, as was previously observed for various HTS's. ${ }^{8-17}$ The decrease of more than a factor of 2 of the oxygen isotope shift $\Delta \lambda_{a b}(0) / \lambda_{a b}(0)$ with increasing the magnetic field from $0.2 \mathrm{~T}$ to $0.6 \mathrm{~T}$ (see Table I) thus implies that ${ }^{16} \lambda_{a b}(0)$ increases stronger with field than ${ }^{18} \lambda_{a b}(0)$, as expected for ${ }^{16} m_{a b}^{*}<{ }^{18} m_{a b}^{*}$ (see discussion above).

In order to study the magnetic-field dependence of $\lambda_{a b}$ in more detail, $\sigma_{s c}$ was measured as a function of $H$ at $T$ $=20 \mathrm{~K}$ for the ${ }^{16} \mathrm{O}$ - and ${ }^{18} \mathrm{O}$-substituted $\mathrm{YBa}_{2} \mathrm{Cu}_{4} \mathrm{O}_{8}$ samples (see Fig. 3). To ensure that the quality of the fit does not depend on the external field, in the inset the normalized $\chi_{\text {norm }}^{2}$ is plotted as a function of $H\left[\chi_{\text {norm }}^{2}=\chi^{2} /(n+\sqrt{2 n})\right.$, where $n$ is the number of degrees of freedom minus one and $2 n$ is the variance of the $\chi^{2}$ distribution]. For both ${ }^{16} \mathrm{O}-$ and ${ }^{18} \mathrm{O}$-substituted samples the values $\chi_{\text {norm }}^{2}$ are very close to each other and almost equal to unity. This implies that in the whole field range the experimental data can be satisfactory fitted with the simple Gaussian line as it states, e.g., in Ref. 23. It is seen (see Fig. 3) that for both samples $\sigma_{s c}$ first increases, goes through a pronounced maximum around $\mu_{0} H \sim 0.2 \mathrm{~T}$, and then decreases. Note that for conventional $s$-wave superconductors, where $\lambda$ is field independent, $\sigma_{s c}$ increases with increasing magnetic field up to $H \simeq 2 H_{c 1}$ and then stays constant for fields much smaller than $H_{c 2}$ (Ref. 23) $\left(H_{c 1}\right.$ and $H_{c 2}$ are the first and second critical fields, respectively). For $d$-wave superconductors, however, $\lambda$ depends on the field. This leads to a decrease of $\sigma_{s c}(H)$ for magnetic fields higher that $2 H_{c 1}$. Such behavior is generally observed for various HTS's. ${ }^{3,21}$ The solid lines represent results of the fits by means of the relation

$$
\frac{\lambda_{a b}^{-2}(B)}{\lambda_{a b}^{-2}(B=0)}=\frac{\sigma_{s c}(B)}{\sigma_{s c}(B=0)}=1-K \sqrt{B},
$$

which takes into account the nonlinear correction to $\lambda_{a b} \cdot{ }^{7,24}$ Here $K$ is the parameter depending on the strength of the

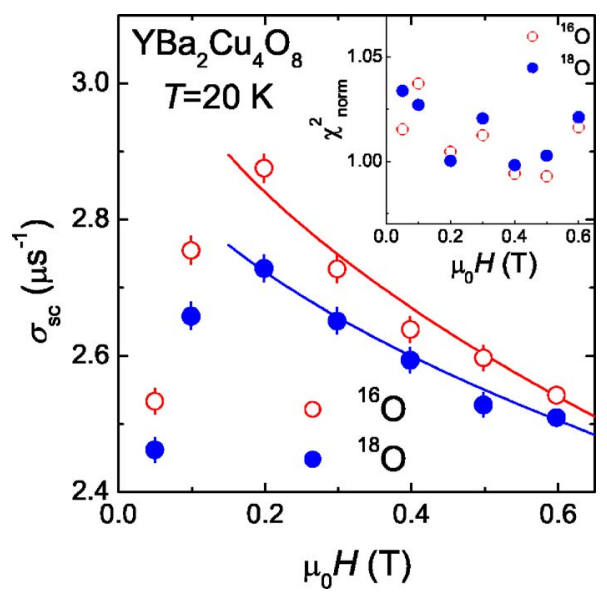

FIG. 3. (Color online) Magnetic-field dependence of the $\mu \mathrm{SR}$ depolarization rate $\sigma_{s c}$ for ${ }^{16} \mathrm{O}-(\bigcirc)$ and ${ }^{18} \mathrm{O}-(\bullet)$ substituted $\mathrm{YBa}_{2} \mathrm{Cu}_{4} \mathrm{O}_{8}$ samples at $T=20 \mathrm{~K}$. Solid curves represent fits of the data using Eq. (2). The inset shows $\chi_{\text {norm }}^{2}$ vs $H . \chi_{\text {norm }}^{2} \simeq 1$ confirms that in the whole field range the experimental data can be satisfactorily fitted with the simple Gaussian line. See text for details. 
nonlinear effect. In the analysis only the points above $0.1 \mathrm{~T}$ were considered. The magnetic field inside the sample was assumed to be equal to the external magnetic field $B$ $\simeq \mu_{0} H_{\text {ext }}$. The fits yield ${ }^{16} \sigma_{s c}(B=0)=3.25(3) \mu \mathrm{s}^{-1},{ }^{16} K$ $=0.282(5) \mathrm{T}^{-1 / 2}$ and ${ }^{18} \sigma_{s c}(B=0)=3.02(3) \mu \mathrm{s}^{-1}, \quad{ }^{18} K$ $=0.220(5) T^{-1 / 2}$ for the ${ }^{16} \mathrm{O}$ - and ${ }^{18} \mathrm{O}$-substituted samples, respectively. The larger ${ }^{16} \sigma_{s c}(B)$ and its stronger field decrease in comparison with ${ }^{18} \sigma_{s c}(B)$ is consistent with the finding ${ }^{18} m_{a b}^{*}>{ }^{16} m_{a b}^{*}$ (Refs. 8-17) and with the statement that the nonlinear and nonlocal corrections to $\lambda_{a b}$ decrease with increasing in-plane supercarrier mass $m_{a b}^{*}$. One should mention, however, that the measurements were performed in magnetic fields well below $H_{c 2}\left(0.0006 \leqslant H / H_{c 2} \leqslant 0.008\right)$, so that it is difficult to draw any firm conclusion about the precise field dependence of $\lambda_{a b}$. In particular, the stronger field dependence of ${ }^{16} \sigma_{s c}$ suggests that at some characteristic field the sign of the OIE on $\lambda_{a b}$ should change. Further investigations at higher fields are needed to clarify this point.

All the above-observed features are consistent with the picture where the mass of the charge carriers $m_{a b}^{*}$ depends on the oxygen isotope mass. It stems from the isotope dependence of the in-plane magnetic penetration depth $\lambda_{a b}$, which was found to be larger for the samples with the heavier oxygen isotope $\left({ }^{18} \lambda_{a b}>{ }^{16} \lambda_{a b}\right)$. An additional confirmation comes from the stronger magnetic-field dependence of $\lambda_{a b}$ in the ${ }^{16} \mathrm{O}$-substituted samples. The latter one is determined by the fact that both the nonlinear and nonlocal corrections to $\lambda_{a b}$, arising from the presence of nodes in the superconducting gap, decrease with increasing $m_{a b}^{*}$.

In conclusion, the magnetic-field dependence of the OIE on the in-plane magnetic-field penetration depth $\lambda_{a b}$ was studied in $\quad \mathrm{YBa}_{2} \mathrm{Cu}_{4} \mathrm{O}_{8}, \quad \mathrm{Y}_{0.8} \mathrm{Pr}_{0.2} \mathrm{Ba}_{2} \mathrm{Cu}_{3} \mathrm{O}_{7-\delta}$, and $\mathrm{Y}_{0.7} \mathrm{Pr}_{0.3} \mathrm{Ba}_{2} \mathrm{Cu}_{3} \mathrm{O}_{7-\delta}$ hole-doped HTS's. As a result $\lambda_{a b}$ for the ${ }^{16} \mathrm{O}$-substituted samples was found to be smaller than for the ${ }^{18} \mathrm{O}$-substituted ones. For all the samples the OIE on $\lambda_{a b}$ decreases by more than a factor of 2 with increasing magnetic field from $\mu_{0} H=0.2 \mathrm{~T}$ to $\mu_{0} H=0.6 \mathrm{~T}$. Both of the above-mentioned effects can be explained by the isotope dependence of the in-plane charge carrier mass $m_{a b}^{*}$.

This work was partly performed at the Swiss Muon Source $(\mathrm{S} \mu \mathrm{S})$, Paul Scherrer Institute (PSI, Switzerland). The authors are grateful to A. Amato and D. Herlach for assistance during the $\mu \mathrm{SR}$ measurements. This work was supported by the Swiss National Science Foundation, the K. Alex Müller Foundation, and in part by the NCCR program MaNEP, the SCOPES Grant No. IB7420-110784, and the EU Project CoMePhS.
*Electronic address: rustem.khasanov@physik.unizh.ch

${ }^{1}$ D. J. Scalapino, Phys. Rep. 250, 329 (1995).

${ }^{2}$ C. C. Tsuei, J. R. Kirtley, C. C. Chi, L. S. Yu-Jahnes, A. Gupta, T. Shaw, J. Z. Sun, and M. B. Ketchen, Phys. Rev. Lett. 73, 593 (1994).

${ }^{3}$ J. E. Sonier, J. H. Brewer, and R. F. Kiefl, Rev. Mod. Phys. 72, 769 (2000).

${ }^{4}$ M. H. S. Amin, M. Franz, and I. Affleck, Phys. Rev. Lett. 84, 5864 (2000).

${ }^{5}$ M. H. S. Amin, Ph.D. thesis, University of British Colombia, 1999; cond-mat/0011455 (unpublished).

${ }^{6}$ G. E. Volovik, JETP Lett. 58, 469 (1993).

${ }^{7}$ H. Won and K. Maki, Europhys. Lett. 54, 248 (2001).

${ }^{8}$ G. M. Zhao and D. E. Morris, Phys. Rev. B 51, 16487 (1995).

${ }^{9}$ R. Khasanov, A. Shengelaya, K. Conder, E. Morenzoni, I. M. Savić, and H. Keller, J. Phys.: Condens. Matter 15, L17 (2003).

${ }^{10}$ R. Khasanov, A. Shengelaya, E. Morenzoni, M. Angst, K. Conder, I. M. Savić, D. Lampakis, E. Liarokapis, A. Tatsi, and H. Keller, Phys. Rev. B 68, 220506(R) (2003).

${ }^{11}$ R. Khasanov, D. G. Eshchenko, H. Luetkens, E. Morenzoni, T. Prokscha, A. Suter, N. Garifianov, M. Mali, J. Roos, K. Conder, and H. Keller, Phys. Rev. Lett. 92, 057602 (2004).

${ }^{12}$ R. Khasanov, A. Shengelaya, E. Morenzoni, K. Conder, I. M. Savić, and H. Keller, J. Phys.: Condens. Matter 16, S4439 (2004).
${ }^{13}$ R. Khasanov, A. Shengelaya, K. Conder, E. Morenzoni, I. M. Savić, J. Karpinski, and H. Keller, Phys. Rev. B 74, 064504 (2006).

${ }^{14}$ G. M. Zhao, M. B. Hunt, H. Keller, and K. A. Müller, Nature (London) 385, 236 (1997).

${ }^{15}$ G. M. Zhao, K. Conder, H. Keller, and K. A. Müller, J. Phys.: Condens. Matter 10, 9055 (1998).

${ }^{16}$ J. Hofer, K. Conder, T. Sasagawa, G.-M. Zhao, M. Willemin, H. Keller, and K. Kishio, Phys. Rev. Lett. 84, 4192 (2000).

${ }^{17}$ G. M. Zhao, V. Kirtikar, and D. E. Morris, Phys. Rev. B 63, 220506(R) (2001).

${ }^{18}$ J. L. Tallon, R. S. Islam, J. Storey, G. V. M. Williams, and J. R. Cooper, Phys. Rev. Lett. 94, 237002 (2005).

${ }^{19}$ J. Karpinski, S. Rusiecki, E. Kaldis, B. Bucher, and E. Jilek, Physica C 160, 449 (1989).

${ }^{20}$ K. Conder, Mater. Sci. Eng., R. 32, 41 (2001).

${ }^{21}$ P. Zimmermann, H. Keller, S. L. Lee, I. M. Savić, M. Warden, D. Zech, R. Cubitt, E. M. Forgan, E. Kaldis, J. Karpinski, and C. Krüger, Phys. Rev. B 52, 541 (1995).

${ }^{22}$ A. T. Fiory, A. F. Hebard, R. H. Eick, P. M. Mankiewich, R. E. Howard, and M. L. O’Malley, Phys. Rev. Lett. 65, 3441 (1990).

${ }^{23}$ E. H. Brandt, Phys. Rev. B 37, 2349 (1988).

${ }^{24}$ I. Vekhter, J. P. Carbotte, and E. J. Nicol, Phys. Rev. B 59, 1417 (1999). 